\title{
LAS SPIN-OFFS ACADÉMICAS Y SU POSIBLE CONFIGURACIÓN COMO EMPRESAS DE ECONOMÍA SOCIAL
}

\author{
POR \\ Carlos VARGAS VASSEROT ${ }^{2}$
}

\section{RESUMEN}

El estudio trata de las posibilidades y limitaciones que tienen las empresas de economía social (cooperativas y sociedades laborales fundamentalmente) para ser utilizadas para la creación de Empresas de Base Tecnológica en el ámbito académico. Primero se realiza una aproximación básica al concepto de la economía social, para posteriormente diferenciar las empresas simplemente innovadoras (start-up en terminología anglosajona) de las empresas que explotan una tecnología o conocimiento transferido por una Universidad y en la que parte de los socios son investigadores de dicha institución (spin-offs académicas). Aunque ambas se engloban en un término amplio de Empresas de Base Tecnológica no son lo mismo. Mientras que para desarrollar empresas de alto y rápido desarrollo tecnológico por egresados y estudiantes universitarios no existe problema alguno para constituirse como empresas de economía social, para hacer una Empresa de Base Tecnológica académica existen determinadas barreras legales. Los investigadores y socios de la empresa suelen ser profesores que trabajan a tiempo completo y en exclusiva en la Universidad, lo que impide, en principio, estar realizando de manera continuada una actividad laboral o de prestación de servicios en una entidad privada, que es el núcleo de la actividad mutualista típica de las sociedades cooperativas y sociedades laborales. Además, si la Universidad participa en el capital social

\footnotetext{
${ }^{1}$ Estudio realizado en el marco del Proyecto de Investigación del MICINN DER2009-08332, sobre "Transferencia de Resultados de Investigación. Identificación de problemas y propuestas de solución”, y del Grupo de Investigación de la Junta de Andalucía SEJ-200 "Derecho Público y Privado de la Agroalimentación y de la Innovación Tecnológica", adscrito al Campus de Excelencia Internacional Agroalimentario (ceiA3).

${ }^{2}$ Profesor Titular de Derecho Mercantil de la Universidad de Almería. Director de la Oficina de Transferencia de Resultados de Investigación de la Universidad de Almería. Correo electronico: cvargas@ual.es

REVESCO No 107 - Primer Cuatrimestre 2012 - ISSN: 1885-8031 - www.ucm.es/info/revesco
}

DOI: $10.5209 /$ rev_REVE.2012.v107.38748

Fecha de recepción: 20/07/2011

Fecha de aceptación: 08/09/2011 
de la spin-off constituida, que es la tendencia, las ventajas de las empresas de economía social no son tan claras para el organismo público que suele preferir ser socio de una sociedad de capital.

Palabras clave: empresas de base tecnológica - cooperativas - Universidad - sociedades laborales - economía social

Claves ECONLIT: P130, I230, I250

\title{
THE ACADEMIC SPIN-OFFS AND SET AS SOCIAL ECONOMY ENTERPRISES
}

\begin{abstract}
The study deals with the possibilities and constraints facing social economy enterprises (co-operatives and worker societies mainly) to be used for creating Spin-off an academic field. First is a basic approach to the concept of social economy, to further differentiate the companies simply innovative (star-up in Anglo-Saxo terminology) of companies operating a technology or knowledge transferred by a university and in which the partners are researchers at that institution (academic spin-offs). Although both fall into a broad term for Spin-off are not the same. While for companies that develop high technology and rapid development of graduates and colleges students doesn't exist any problem to establish itself as social economy enterprises, to constitute a formal academic spin-off there are certain legal barriers. Researchers and business associates are often teachers who work full time and exclusively at the university, which prevents, in principle, be continuously performing a work activity or the provision of services in a private entity, which is the core of typical mutual activity of co-operatives and worker societies. Furthermore, if the University participates in the capital of the spin-off constituted, which is the tendency, the benefits of social economy enterprises are not as clear for the public organism which usually prefers to be a partner in a capital company.
\end{abstract}

Keywords: spin-off - co-operatives -University- worker societies - social economy 


\section{EL EMPRENDIMIENTO UNIVERSITARIO EN EL MARCO DE LA ECONOMÍA SOCIAL}

Aunque el concepto de economía social es difícil de concretar, ya que constituye una realidad muy diversa, generalmente se entiende por tal, toda actividad económica de carácter privado basada en la asociación de personas en entidades de tipo democrático y participativo, con primacía de las aportaciones personales sobre las de capital y donde la distribución del beneficio y la toma de decisiones no están directamente ligadas con el capital aportado por cada socio.

En España recientemente se ha promulgado la Ley 5/2011 de Economía Social (LES), que tiene por objeto establecer un marco jurídico común para el conjunto de entidades que integran la economía social, con pleno respeto a la normativa específica aplicable a cada una de ellas, así como determinar las medidas de fomento a favor de las mismas en consideración a los fines y principios que les son propios. La LES denomina como economía social al conjunto de las actividades económicas y empresariales, que en el ámbito privado llevan a cabo aquellas entidades que, de conformidad con una serie de principios extraídos de los cooperativos, persigan bien el interés colectivo de sus integrantes, bien el interés general económico o social, o ambos ${ }^{3}$. Por tanto, ya desde la definición legal de Economía Social, se hace mención a los principios orientadores de la economía social lo que hace necesario hacer un breve repaso de cuáles son estos principios, que vienen contenidos en su artículo 4.

a. Primacía de las personas y del fin social sobre el capital, que se concreta en gestión autónoma y transparente, democrática y participativa, que lleva a priorizar la toma de decisiones más en función de las personas y sus aportaciones de trabajo y servicios prestados a la entidad o en función del fin social, que en relación a sus aportaciones al capital social.

b. Aplicación de los resultados obtenidos de la actividad económica principalmente en función del trabajo aportado y servicio o actividad realizada por las socias y socios o por sus miembros y, en su caso, al fin social objeto de la entidad.

\footnotetext{
${ }^{3}$ PANIAGUA ZURERA, M. Las empresas de la economía social más allá del comentario a la Ley 5/2011, de economía social. Madrid: Marcial Pons, 2011, passim.
} 
c. Promoción de la solidaridad interna y con la sociedad que favorezca el compromiso con el desarrollo local, la igualdad de oportunidades entre hombres y mujeres, la cohesión social, la inserción de personas en riesgo de exclusión social, la generación de empleo estable y de calidad, la conciliación de la vida personal, familiar y laboral y la sostenibilidad.

d. Independencia respecto a los poderes públicos.

Estos principio tienen su origen en la Carta de Principios de la Economía Social, aprobada en 2002 por la Conferencia Europea Permanente de Cooperativas, Mutualidades, Asociaciones y Fundaciones (CEP-CMAF), plataforma europea representativa de las cuatro familias de entidades de la Economía Social, hoy denominada Social Economy Europe. En ella se señala las características comunes de las diversas formas jurídicas de las organizaciones de Economía Social, que tienen su antecedente más remoto en los principios cooperativos. Recordemos que éstos son elementos distintivos de las cooperativas que formula y reformula la Alianza Cooperativa Internacional (ACI) cada cierto tiempo, en el marco de algunos de los Congresos Mundiales de dicha organización (París 1937; Viena 1966 y Manchester 1995), para adaptarlos a las nuevas circunstancias económicas y sociales, pero sin perder nunca de vista la esencia de los principios formulados por la experiencia de Rochdale.

En el listado de entidades de la economía social que contiene el artículo 5.1 de la LES, se incluyen a las cooperativas, las mutualidades, las fundaciones y las asociaciones que lleven a cabo actividad económica, las sociedades laborales, las empresas de inserción, los centros especiales de empleo, las cofradías de pescadores y las Sociedades Agrarias de Transformación. De todas estas entidades de economía social nos centraremos en las cooperativas y sociedades laborales, tipos sociales que parecen adecuarse mejor como vestidura jurídica para constituir una empresa para explotar la tecnología o el conocimiento surgido en el seno de un organismo público de investigación y del que forman parte, como socios, investigadores adscritos a dicho centro y, en ocasiones, la propia entidad.

La constitución o participación en sociedades por parte de las Universidades y otros organimos públicos de investigación alcanza verdadero interés, con peculiaridades jurídicas propias, cuando se utilizan como mecanismo de explotación de los resultados de investigación que se obtengan en su seno. En España, como ocurre en la mayoría de países iberoamericanos es más común el término de empresa de base tecnológica (EBT), con lo que se focaliza en el 
carácter tecnológico del proyecto empresarial. Lo que ocurre es que el término de EBT se ha generalizado y a veces con esta acepción se califican a empresas simplemente innovadoras cuyos resultados tecnológicos supongan un avance en la obtención de nuevos productos, procesos o servicios, o la mejora sustancial de otros ya existentes, lo que se asemeja con el término anglosajón de start-up.

Incluso en el ámbito universitario se discute la propiedad del término de EBT, en cuanto que parece excluir a las empresa cuya base sea la explotación científica de un conocimiento científico en otras áreas de investigación distintas a las puramente tecnológicas. Es de destacar que en la reciente tramitación de la Ley de la Ciencia, la Tecnología y la Innovación, hasta el último momento (enmienda número 144 retirada el 28 de abril de 2011) se discutió si se aceptaba o no la enmienda. Se debatió si se sustituía la denominación de empresa de base tecnológica por la de empresas basadas en el conocimiento para dar así cabida a iniciativas empresariales surgidas de investigaciones en el campos de las Ciencias Sociales y Humanidades como pueden ser las jurídicas y económicas, que con una interpretación restrictiva del concepto de tecnología quedan fuera del concepto de EBT universitaria.

El otro término comunmente utilizado para referirse a esta realidad empresarial es el de spin-off, que hace referencia al proceso de creación de una sociedad subdisiaria en el seno de una organización matriz. En el ámbito universitario, junto a las spin-offs creadas con la participación activa de profesores e investigadores que trabajan en la institución, tenemos a las empresas constituidas por egresados o estudiantes de la Universidad con apoyo de ésta (graduate spin-off) pero sin ninguna vinculación, ni tecnológica ni laboral con la institución académica distinta a la participación de programas de apoyo a su constitución como empresas y en las que no hay ningún obstaculo jurídico para utilizar empresas de economía social. Es más, considero que es muy recomendable el uso de empresas participativas y en las que rigen un ideal de democracia interna y que están tan ligadas al autoempleo. Y con ese ideario han nacido algunas de las mas importantes empresas tecnológicas del mundo en un momento embrionario formada por estudiantes o egresados universitarios: Hewlett Packard o Apple (aunque Steve Jobs, como reconoció en el famoso discurso en la Universidad de Stanford durante la apertura del curso de 2005, abandonó pronto los estudios universitarios), etc. 
Pero las cosas son bien distintas cuando de lo que se trata es de crear una empresa conformada por profesores e investigadores universitarios para explotar los resultados de sus investigaciones y cumplir con la función de transferencia 4, puesto que existen varios obstaculos legales para las utilización de sociedades cooperativas o laborales. En este caso, el término spin-off refleja mejor que el de EBT el necesario proceso de disgregación de la entidad matriz (léase Universidad) y de transferencia de tecnología o conocimiento a la nueva empresa que se crea, pero también es cierto que el de empresa de base tecnológica hace referencia a una parte fundamental de este emprendimiento universitario, como es la explotación comercial de nuevas líneas de productos o de servicios basados en el desarrollo de tecnologías o conocimientos científicos-tecnológicos.

Pero antes de tratar las limitaciones y obstaculos legales que hemos encontrado para utilizar estos tipos sociales para crear Empresas de Base Tecnológica o spin-offs académicas, vamor a hacer un breve recorrido por el marco legal de esta forma de articular la transferencia de resultados de investigación que nace de organismos públicos de investigación ${ }^{5}$.

\section{EL MARCO LEGAL DE LAS SPIN-OFFS ACADÉMICAS EN ESPAÑA}

\subsection{Las Empresas de Base Tecnológica en la Ley Orgánica de Universidades}

Aunque antes ya había habido experiencias de este tipo, se puede señalar la Ley Orgánica 6/2001 de Universidades (LOU) como la primera norma que regula el fenómeno en nuestro país. En dicha norma, se sientan las bases para la creación de EBT como instrumento para difundir y explotar los resultados de investigación generados en la Universidad. En concreto, su artículo 41.2, letra g) in fine, después de reconocer la necesaria vinculación entre la investigación universitaria y el sistema productivo como vía para articular la transferencia de los conocimientos generados y la presencia de la Universidad en el proceso de innovación

\footnotetext{
${ }^{4}$ Sobre esta tercera función universitaria, VALLE PASCUAL, J.M. y MARTÍNEZ FERNÁNDEZ, S. La transferencia del conocimiento, ¿una nueva función social universitaria? Medios personales y materiales. En Bases jurídicas para la gestión universitaria, t. I. Alicante: Universidad de Alicante, 2008, p. 285-316. De interés, con un interesante estudio del origen histórico de las Universidades, SOSA WAGNER, F. El mito de la autonomía universitaria. Madrid, 2007, p. 17-50. Con una visión crítica de los peligros de mercantilización de la ciencia, SlAUGther, S. y LESLIE, L.L. Academic Capitalist. Politics, Policies and the Entrepreneurial University. Baltimore: The Johns Hopkins, 1997.

${ }^{5}$ Con mucho más detalle sobre este marco legal, remitimos a VARGAS VASSEROT, C. Las empresas de base tecnológica como instrumento de transferencia de resultados de invesrtigación. En Estudios de Derecho Mercantil en homenaje al Profesor José María Muñoz Planas. Madrid: Civitas, 2011, p. 857-876.
} 
del sistema productivo y de las empresas, establecía que "dicha vinculación podrá, en su caso, llevarse a cabo a través de la creación de Empresas de Base Tecnológica a partir de la actividad universitaria, en cuyas actividades podrá participar el personal docente e investigador de las Universidades conforme al régimen previsto en el artículo 83”.

Como se puede observar, la atención que la LOU en su versión original dedicaba a las EBT era muy escasa, y prácticamente se limitaba a reconocer que las Universidades podían crear empresas a partir de la "actividad universitaria" como mecanismo de transferencia, posibilidad que ya estaba implícita en el artículo 84 de la Ley que establecía que las Universidades, para la "promoción y desarrollo de sus fines" [y el artículo 1.2, letra c) incluía a la transferencia de resultados de investigación como función de las mismas] podían crear, por sí solas o en colaboración con otras entidades, empresas, fundaciones u otras personas jurídicas de acuerdo con la legislación general aplicable ${ }^{6}$.

Por ello hay que reconocer a la Ley Orgánica 4/2007 que modifica la LOU (LOMLOU) como la verdadera artífice del impulso dado a la creación de EBT en España en los últimos años, a pesar de contener una regulación sobre este tipo de empresa compleja, dispersa y, sobre todo, inacabada. El artículo central de la LOU dedicado originalmente a las EBT queda prácticamente inalterado [art. 41.2, letra g)], pero se añade un apartado 3 al artículo 83 que contempla la posibilidad de incoporación, mediante una excedencia temporal del profesorado a una Empresa de Base Tecnológica cuando cumpla determinadas condiciones: "creada o desarrollada a partir de patentes o de resultados generados por proyectos de investigación financiados total o parcialmente con fondos públicos y realizados en Universidades".

A esto hay que añadir la importante disposición adicional vigésimo cuarta, introducida a última hora en el Senado, que preveía la no aplicación de ciertas limitaciones contenidas en la Ley 53/1984 de incompatibilidades del personal al servicio de las Administraciones públicas [participación en el órgano de administración -art.12.1, letra b) - ; posesión de más de un $10 \%$ del capital social -art. 12.1, letra d)] a los profesores funcionarios de los cuerpos

\footnotetext{
${ }^{6}$ Como puso de manifiesto la doctrina que analizó el texto de la LOU original en materia de transferencia, seguían existiendo practicamente las mismas limitaciones legales que había en nuestro ordenamiento antes de la promulgación de la Ley para el desarrollo efectivo de esta tercera misión de la Universidad: por todos, GÓMEZ OTERO, C. y VALLE PASCUAL, J.M. Si el legislador nos echara una mano. En IV Seminario sobre aspectos jurídicos de la gestión universitaria. Girona, 2001.
} 
docentes universitarios cuando participen en empresas de base tecnológica que cumplan una serie de condiciones: a) sean promovidas por su Universidad y participadas por ésta o por alguno de los entes previstos en el artículo $84 \mathrm{LOU}$; b) sean creadas a partir de patentes o de resultados generados por proyectos de investigación realizados en universidades; y c) se cumplan una serie de requisitos procedimentales (acuerdo explícito del Consejo de Gobierno que certifique la naturaleza de base tecnológica de la empresa).

La disposición adicional 24a terminaba diciendo que "el Gobierno regulará las condiciones para la determinación de la naturaleza de base tecnológica" de estas empresas. Algo que aún, cuatro años después, no se ha hecho. La mayor discusión que se planteó con el contenido de este precepto era si resultaba necesario esperar a la promulgación de la anunciada normativa de desarrollo (hasta seis versiones del Real Decreto regulador circularon por distintos foros) para poder aplicar el régimen de excedencia y la aplicación de las excepciones a la Ley de incompatibilidades que establecía la LOMLOU o si, por el contrario, con lo dispuesto en el texto de Ley y, en concreto en dicha disposición adicional $24^{\mathrm{a}}$, era suficiente para su aplicación directa a las EBT creadas por las Universidades.

Lo cierto es que un buen número de Universidades españolas dieron un paso adelante y diseñaron y muchas veces aprobaron a través de sus Consejos de Gobierno Reglamentos para la creación de EBT: Por poner algunos de los muchos ejemplos de estas normativas internas: Reglamento de Empresas de Base Tecnológica de la Universidad de Almería (31 de octubre de 2008); Reglamento de creación de empresas de base tecnológica y spin-offs de la Universidad Autónoma de Barcelona (7 de octubre de 2009); Reglamento de constitución, incorporación y funcionamiento de las Empresas de Base Tecnológica de la Universidad de Murcia (13 de febrero de 2009); Reglamento de EBT, EBNT y Empresas asociadas de la Universidad de León (20 de diciembre de 2010).

Al final, estos Reglamentos o normas internas de las Universidades regularon lo que no terminó de decir el legislador: concepto de EBT, procedimiento de constitución, régimen de participación en el capital social por parte de la Universidad, contenido del contrato de transferencia, contenido del acuerdo de socios y un largo etcétera de cuestiones jurídicas que habían quedado en el aire. En este contexto, de gran inseguridad jurídica (cada Universidad ha diseñado su particular modelo), en el que, como tantas veces ocurre, los hechos corrieron más rápido que el legislador, se publicó primero la LES y poco después la LCTI y ambas normas 
insisten en la importancia e interés de que las Universidades participen en sociedades mercantiles para transferir y explotar conocimiento de su titularidad.

\subsection{Las Empresas Innovadoras de Base Tecnológica de la Ley de Economía Sostenible}

La Ley 2/2011 de Economía Sostenible (LES) establece en su artículo 56, titulado Cooperación de los agentes públicos de ejecución con el sector privado a través de la participación en Empresas Innovadoras de Base Tecnológica, que los Organismos Públicos de Investigación, incluídas las Universidades, podrán participar en el capital de sociedades mercantiles cuyo objeto social sea la realización de alguna de las siguientes actividades: a) La investigación, el desarrollo o la innovación; b) La realización de pruebas de concepto; c) La explotación de patentes de invención y, en general, la cesión y explotación de los derechos de la propiedad industrial e intelectual; d) El uso y el aprovechamiento, industrial o comercial, de las innovaciones, de los conocimientos científicos y de los resultados obtenidos y desarrollados por dichos agentes; e) La prestación de servicios técnicos relacionados con sus fines propios.

Más adelante, el artículo 64.2 de la LES señala que la colaboración entre las universidades y el sector productivo podrá articularse mediante cualquier instrumento admitido por el ordenamiento jurídico y, en particular, mediante la constitución de empresas innovadoras de base tecnológica (letra a) ${ }^{7}$. Y en su número 3 , dispone que las universidades podrán promover la creación de empresas innovadoras de base tecnológica, abiertas a la participación en su capital societario de uno o varios de sus investigadores, al objeto de realizar la explotación económica de resultados de investigación y desarrollo obtenidos por éstos.

Pero realmente todo esto ya estaba permitido, al menos para las Universidades, por el contenido del artículo 84 de la LOU en su versión original de 2001, que para la promoción y desarrollo de sus fines, las Universidades, con la aprobación del Consejo Social, pueden crear,

\footnotetext{
${ }^{7}$ A la novedad de la terminología utilizada por la LES, que habla de empresas innovadoras de base tecnológica y no simplemene de empresas de base tecnológica, como hacía la LOU y hizo después la LCTI, se refiere PETIT LAVALL, M. ${ }^{\text {a } V . ~ E l ~ a n t e p r o y e c t o ~ d e ~ L e y ~ d e ~ E c o n o m i ́ a ~ S o s t e n i b l e ~ y ~ l a ~ t r a n s f e r e n c i a ~ d e ~ r e s u l t a d o s ~ d e ~ l a ~ a c t i v i d a d ~}$ investigadora de las Universidades públicas mediante la constitución de Empresas de Base Tecnológica (EBTS). En GÓMEZ-SEGADE y GARCÍA VIDAL. El Derecho Mercantil en el umbral del siglo XXI. Libro homenaje Fernández-Novo. Madrid, 2010.
} 
por sí solas o en colaboración con otras entidades públicas o privadas, empresas, léase sociedades, de acuerdo con la legislación general aplicable, por lo que las novedades de la LES en esta materia es más una declaración de intenciones que un cambio normativo.

En lo que resulta muy interesante la LES, es la preocupación que manifiesta por aclarar la titularidad y carácter patrimonial de los resultados de investigación desarrollados por investigadores de los Organismos Públicos de Investigación (art. 54) y por exigir la debida articulación jurídica y el sometimiento a ciertas reglas la transmisión a terceros de estos derechos sobre los resultados de la actividad investigadora (arts. 55). Y no se puede olvidar, y esto me parece fundamental, que el conocimiento que se explota en la EBT, al tener su origen en la Universidad, es de titularidad universitaria y se hace necesaria la suscripción de un contrato de transferencia de tecnología que ceda este conocimiento ${ }^{8}$. Y esto mismo, es aplicable a las invenciones que se desarrollen por los investigadores socios de las EBT ya constituidas. Así, a menos que exista un contrato o proyecto de investigación que acuerde la cesión de los derechos de propiedad industrial, las invenciones cuyos inventores sean profesores universitarios son de titularidad universitaria (cfr. art. 20 Ley de Patentes de Invención), aunque sean socios de las spin-off. Por ello es tan importante articular adecuadamente la transferencia de los resultados de investigación que se pretenden explotar por la EBT.

\subsection{El impulso de la participación de los organismos públicos de investigación en sociedades mercantiles en la Ley de la Ciencia, la Tecnología y la Innovación}

La Ley 11/2011 de la Ciencia, la Tecnología y la Innovación (LCTI), de 1 de junio de 2011, en su artículo 18 regula el régimen de autorización para prestar servicios en sociedades mercantiles en una actuación relacionada con las prioridades científico técnicas establecidas en la Estrategia Española de Ciencia y Tecnología o en la Estrategia Española de Innovación y la excepción al régimen de incompatibilidades contenido en los apartados b) y d) del artículo 12.1 de la Ley 53/1984. Después, insiste de nuevo en la necesidad de impulsar la participación de los Organismos Públicos de Investigación en sociedades mercantiles tanto

\footnotetext{
${ }^{8}$ Un caso de interés, donde se expone la problemática que genera la falta de transferencia de ese conocimiento, lo podemos ver en VARGAS VASSEROT, C. VARGAS VASSEROT, C. La titularidad de las patentes universitarias: el caso Gatorade. Uciencia, no 5, 2010, p. 14 y s.
} 
como mecanismo para la valorización del conocimiento [art. 33.1, letra c)] como para la transferencia del mismo [art. 33.1, letra d) $]^{9}$.

Por otra parte la LCTI, modifica varios precepto de la LOU, para adaptarla a la nueva normativa (art. 80.5, art. 84, disp. Adicional 10. ${ }^{\mathrm{a}} 1$ bis), pero en cambio no deroga la disposición adicional 26. ${ }^{\mathrm{a}}$-como sí estaba inicialmente previsto en el proyecto de Ley-, con lo que queda vigente la obligación del Gobierno de regular las condiciones para la determinación de la naturaleza de base tecnológica.

Por último, cabe indicar que la LCTI ha regulado una nueva figura empresarial, que es la la denominada Joven Empresa Innovadora (JEI). Pues bien, según dispone la disposición adicional tercera, el Ministerio de Ciencia e Innovación otorgará dicha condición a aquella empresa que: tenga una antigüedad inferior a 6 años; que haya realizado unos gastos en investigación, desarrollo e innovación tecnológica que representen al menos el 15\% de los gastos totales de la empresa durante los dos ejercicios anteriores; y que se haya constatado, mediante una evaluación de expertos que la empresa desarrollará productos, servicios o procesos tecnológicamente novedosos o sustancialmente mejorados con respecto al estado tecnológico actual del sector correspondiente y que comporten riesgos tecnológicos o industriales. Dicha disposición establece que el Gobierno, en el plazo de un año tras la entrada en vigor de la LCTI, aprobará su Estatuto Legal, inspirado en experiencias europeas de éxito, como aspecto clave para el apoyo de sociedades de reciente creación que dedican una parte significativa de su facturación a actividades de I+D+i. Muchas EBT, spin-offs académicas o de egresados universitarios, seguro que cumplen con los requisitos de estas Jovenes Empresas Innovadoras y podrán acogerse a los programas de incentivos que se supone que se van a desarrollar en breve. Y aquí sí que no hay ningún obstaculo legal para que una empresa de Economía Social, siempre que cumpla los requisitos apuntados, se califique como Jovén Empresa Innovadora.

\footnotetext{
${ }^{9}$ Una visión conjunta de ambas normas puede verse en COSTAS DEL PORTAL, I. y OURO FUENTE, A. Participación de los investigadores en los resultados de la investigación. El entorno legal: proyectos de Ley de la Ciencia, la Tecnología y la Innovación y de la Ley de Economía Sostenible. La Ley, nº 7615, 2011, p. 1-15.
} 


\section{LIMITACIONES LEgALES PARA LA UTILIZACIÓN DE EMPRESAS DE ECONOMIA SOCIAL PARA EXPLOTAR CONOCIMIENTO O TECNOLOGÍA DE ORIGEN UNIVERSITARIO}

\subsection{Interpretación amplía del término de sociedad mercantil utilizada por la normativa científica}

A pesar de que la Ley de Economía Sostenible y la Ley de la Ciencia, Tecnología e Innovación hablan en varias ocasiones de la creación de sociedades mercantiles como mecanismo de transferencia de resultados de investigación, considero que esto no es un obstaculo para el uso de cooperativas o sociedades laborales con tal fin. Respecto al carácter mercantil de las sociedades laborales no hay duda alguna, puesto que deriva de su forma, como establece para todas las sociedades de capital (anónimas y de responsabilidad limitada) la Ley de Sociedades de Capital (art. 2). Y para las cooperativas, en mi opinión, tampoco, puesto que el sector cooperativo ha experimentado una galopante evolución en el sentido de progresiva atracción al campo de aplicación del Derecho mercantil, y ya apenas se duda del carácter empresarial, y por tanto mercantil, de las cooperativas. Conviene recordar que no es el lucro el concepto clave para hablar de mercantilidad, sino el de empresa y empresario, y para ambos basta economicidad de la gestión, es decir, sobre la base de un cálculo racional de costes e ingresos; y que vaya dirigida al mercado, en cuanto que la actividad de empresa se proyecta como una actividad de oferta de bienes y servicios en el mercado.

En este sentido me parece más acertada la redacción que tenía, y conserva, la LOU que en su artículo 84 dispone que "para la promoción y desarrollo de sus fines, las Universidades, por sí solas o en colaboración con otras entidades públicas o privadas, y con la aprobación del Consejo Social, podrán crear empresas, fundaciones u otras personas jurídicas de acuerdo con la legislación general aplicable", sin hablar de sociedades mercantiles ${ }^{10}$.

\footnotetext{
${ }^{10}$ Una valoración crítica al contenido de la LOU en esta materia puede verse en Como puso de manifiesto la doctrina que analizó el texto de la LOU original en materia de transferencia, seguían existiendo practicamente las mismas limitaciones legales que había en nuestro ordenamiento antes de la promulgación de la Ley para el desarrollo efectivo de esta tercera misión de la Universidad: por todos,

CAYUELA LÓPEZ, M.J. y PLANAS YANES, G. Comentario art. 41. En Un paseo por la LOU. Análisis sistemático de la Ley Orgánica 6/2001, de 21 de diciembre de Universidades. Madrid, 2003, p. 390 y ss.
}

REVESCO No 107 - Primer Cuatrimestre 2012 - ISSN: 1885-8031 - www.ucm.es/info/revesco 


\subsection{La problemática que plantea simultanear la prestación de trabajo en la Universidad y en la spin-off académica}

El mayor obstaculo para la utilización de tipos sociales de economía social es la necesidad, o al menos conveniencia, de simultaner una actividad científica en el organismo público de investigación y en la spin-off constituida. Si el profesor o investigador no trabaja en la Universidad con dedicación exclusiva, por ejemplo está a tiempo parcial o simplemente es miembro de un grupo de investigación sin relación contractual con la Universidad, en principio no hay problema para simultanear las dos actividades en el ámbito público y en el privado. Pero digo en principio, independientemente del tipo de relación que tenga con la Universidad, ésta puede imponer determinadas obligaciones de confidencialidad o de uso exclusivo de lo que se investiga y descubre en su seno.

La clave en estos casos está en cómo se puede articular la participación activa del investigador en la empresa. Es decir, como trabaja el investigador en la spin-off constituida para explotar los resultados de sus investigación. Y esto para las empresas de economía social es básico, puesto que la esencia de estas empresas es su carácter participativo, que se traduce en la prestación de trabajo, sea una relación laboral o asimiladas. En las Cooperativas de Trabajo Asociado esa prestación laboral es el fundamento de la actividad cooperativizada y en el resto de clases de cooperativas, lo normal es que la participación del investigador en la EBT sea aportando trabajo en calidad de socio laboral. Por su parte, en las Sociedades Laborales esa aportación de trabajo es el fundamento para formar parte de la clase laboral que debe controlar, políticamente hablando, la empresa. Recordemos que el propio concepto de sociedad laboral contenido en el artículo 1 de la Ley de Sociedades Laborales establece que las “sociedades anónimas o de responsabilidad limitada en las que la mayoría del capital social sea propiedad de trabajadores que presten en ellas servicios retribuidos en forma personal y directa, cuya relación laboral lo sea por tiempo indefinido", pueden obtener dicha calificación sin concurren una serie de requisitos.

Pero, de un lado, nada impide que un profesor que no esté en exclusiva pueda desarrollar una actividad laboral en una spin-off académica. Ejemplo de esto es que el XX Premio Arco Iris del Consejo Andaluz de Cooperación en la modalidad de Mejor Cooperativa Innovadora (2009) fue para una EBT de la Universidad de Sevilla dedicada a la gestión 
medioambiental y a la mineralogía aplicada en la que participaban como socios cooperadores profesores a tiempo parcial y egresados de dicha Universidad.

Hasta ahora la principal manera de instrumentar la participación activa de los socios tecnológicos en las spin-offs constituidas era suscribiendo contratos de investigación con la Universidad, también llamados contratos OTRI (art. 83 LOU), para la prestación de servicios, realización de investigaciones o labores de asesoría por parte del profesor, socio a su vez de la empresa. Esta autocontratación, excepto en caso especiales de conflicto de intereses por los miembros del órgano de admnistración, no está prohibida, con la salvaguarda de que la realización de una asesoría remunerada de carácter permanente puede considerarse un trabajo encubierto, lo que va también en contra de la Ley de Incompatibilidades. De esta manera, cabe la posibilidad de que la relación de trabajo del socio con la cooperativa que, como explica el artículo 80 de la LCOOP y concordantes autonómicos para las cooperativas de trabajo asociado, es una relación societaria, se artícule a través contratos de investigación y convenios de colaboración con la Universidad. En tal caso se podría diseñar una cooperativa con socios cooperadores con contratos de trabajo y socios cooperadores sin esa realción laboral de carácter permanente. En cambio, en las Sociedades Laborales, al exigirse que la unión del socio laboral con la sociedad sea a través de servicios retribuidos y por tiempo indefinido, no cabe esta posibilidad para los socios de la clase general (art. 6.1)

Otra opción es pedir una excedencia especial para incorporarse plenamente a la EBT, pero es muy excepcional acudir a esta vía para dejar la Universidad y embarcarte en un proyecto empresarial arriesgado e innovador. A mí no me consta que se haya solicitado ninguna excedencia de este tipo prevista para estos casos en la LOU (art. 83.3 tras la reforma de 2007) ni supongo que se soliciten muchas de las que regula la LCTI (arts. 17.3 y 4), y si se ha hecho seguramente han concurrido determinadas circunstancias personales y profesionales (mejor retribución en la empresa, malestar en la institución, cansancio, ganas de cambiar de trabajo, imposibilidad de compatibilizar horarios, etc.).

En este punto, sí aporta una importante novedad el artículo 18 LCTI, que abre una puerta a una posible participación de los investigadores a tiempo completo en las Univesidades españolas como socios trabajadores en spin-off configuradas como empresas de economía social. Este precepto comprende dos medidas diferentes para incentivar la movilidad y la participación de investigadores en proyectos empresariales surgidos en el 
entorno de organismos públicos de investigación. Por un lado, la autorización para prestar servicios en determinadas sociedades mercantiles y, por otro, la exención parcial del régimen de incompatibilidades a los investigadores que participen en dichos proyectos empresariales.

Ambas medidas se hace depender de que Universidad sea socia ("creadas $o$ participadas por la entidad") de la sociedad mercantil y que la participación del personal investigador sea "una actuación relacionada con las prioridades científico técnicas establecidas en la Estrategia Española de Ciencia y Tecnología o en la Estrategia Española de Innovación”.

Aunque aún no se han desarrollado plenamente dichas estrategias y no se sabe con certeza cuáles son esas prioridades, no es difícil imaginar que algunas de éstas coincidirán con la actividad desarrollada por un buen número de spin-offs académicas. De este modo, en caso de que una EBT universitaria sea a su vez una de estas empresas de carácter estratégico, debemos integrar el contenido de la LCTI (art. 18) y de la LOMLOU (disp. adic. 24. ${ }^{\text {a }}$ ). Y a su vez, si la Universidad participa en una de estas empresas estratégicas pero no cumple los requisitos para ser EBT o no se ha seguido el procedimiento marcado por la LOU y desarrollado por muchos Reglamentos universitarios de EBT (no sean creadas a partir de resultados generados en proyectos de investigación financiados con fondos públicos, no se siga el procedimiento peculiar de constitución o no se certifique por acuerdo de Consejo de Gobierno la naturaleza de base tecnológica, etc.) se aplicará sólo el régimen de la LCTI.

En concreto, el artículo 18, en su apartado $1 .^{\circ}$ y $2 .^{\circ}$ establece que las Universidades podrán autorizar (no es un derecho del investigador) al personal investigador de la institución que justifique motivadamente su participación en una actuación relacionada con determinadas prioridades científico técnicas, la compatibilidad laboral para la prestación de servicios en la spin-off y en la institución. Esta prestación de servicios en la empresa debe articularse mediante un contrato laboral de tiempo parcial y de duración determinada y los reconocimientos de compatibilidad no podrán modificar la jornada ni el horario del puesto de trabajo inicial del interesado en la Universidad.

La redacción del artículo plantea muchas dudas. En cuanto a compatibilizar el trabajos público (Universidad) y privado (spin-off), no queda claro si esto exige o no un cambio en la dedicación profesional en el OPI al que pertenece. Me explico. Si este precepto sólo significa que los profesores en exclusiva (catedráticos, titulares, contratados doctores, ayudantes, 
contratados laborales, etc.) pueden solicitar una reducción de su dedicación y realizar una actividad laboral en la empresa privadas, no parece cambiar nada de lo que pasaba con anterioridad a su promulgación.

En mi opinión esta medida, como mecanismo efectivo de incentivar la participación de los investigadores en sociedades mercantiles, sólo tiene justificación si al investigador se le da la posibilidad de mantenerla relación laboral y el salario de profesor universitario a tiempo completo y dedicación exclusiva y adicionar al mismo el sueldo que reciba como trabajador de la spin-off. Esta opinión se justifica en determinadas expresiones del propio artículo 18 (no se modifica ni la jornada ni el horario en la institución o la posible exención del límite tributario del artículo 16 LISAP) y en que la Universidad, que es la que autoriza dicha compatibilidad, es social de la empresa y puede estar interesada, económicamente hablando, en el desarrollo de la actividad científica y laboral de su investigador en la spin-off constituida. En tal caso, de tener la autorización de la Universidad, nada impide a que el investigador pueda ser socio trabajador en una sociedad cooperativa o socio laboral en una sociedad laboral.

\subsection{La participación de la Universidad como socio de la EBT y limitaciones para hacerlo en empresas de economía social}

De la participación de la Universidad en el capital social depende de que los investigadores puedan gozar del estatus especial que el legislador ha articulado para incentivar la constitución de empresas de este tipo y que se pueden concretar en tres medidas, como es la exención del régimen general de incompatibilidades y posibilidad de compatibilizar el trabajo en la Universidad y en las empresa constituida.

a) Exención del régimen de incompatibilidades

La Ley 53/1984 establece unas serie de incompatibilidades para la participación del personal al servicio de las Admnistraciones Públicas en proyectos empresariales directamente relacionados con las actividades que gestione el departamento, organismos o entidad en la que en las que presten sus servicios. 
La disposición adicional vigésimo cuarta de la LOMLOU preveía la no aplicación de ciertas limitaciones contenidas en la Ley 53/1984 de incompatibilidades del personal al servicio de las Administraciones públicas [participación en el órgano de administración art.12.1, letra b) -; posesión de más de un $10 \%$ del capital social -art. 12.1, letra d)] a los profesores funcionarios de los cuerpos docentes universitarios cuando participen en empresas de base tecnológica promovidas por su Universidad y participadas por ésta o por alguno de los entes previstos en el artículo 84 LOU.

b) Reconocimiento de compatibilidad laboral

Como hemos visto, el artículo 18 LCTI establece que las Universidades públicas podrán autorizar a su personal investigador a la prestación de servicios mediante un contrato laboral a tiempo parcial y de duración en sociedades mercantiles creadas o participadas por la Universidad cuando la actuación del investigador en la empresa esté relacionada con las prioridades científicos técnicas previamente establecidas a nivel nacional. Al personal investigador que preste sus servicios en estas sociedades, también le son de aplicación las excepciones al régimen de incompatibilidad explicadas con anterioridad.

Por otra parte, con la participación en el capital social de las EBT, la Universidad se beneficia de diferentes formas. Una patrimonial, en el sentido de que al ser accionista de varias empresas logra una posible vía de financiación alternativa en caso de éxito empresarial de alguna de ellas, tanto vía reparto de beneficios como por la posible venta de la empresa, Hay ejemplos de venta de spin-off a multinacionales de determinados sectores que han significado cuantiosos ingresos para la Universidad origen de la empresa. Por otra parte, la Universidad consigue facilitar la creación de empresas por parte de sus miembros al levantar alguna de las más importantes limitaciones que pesan sobre ellos en la Ley de incompatibilidades. Por último, cabe indicar que también logra mejorar en su política de transferencia y en un indicador que cada vez se valora más, como es en el de spin-off participadas por la institución. Como hemos visto la importancia de esta participación ha tenido reflejo tanto en la LES como en la LCTI.

Lo que queda claro con la promulgación de la LCTI es que la clara apuesta por incentivar las EBT participadas por el organismo público de investigación, es decir, en las que la Universidad es socia de pleno derecho de la sociedad constituida. En tal caso, junto al 
necesario contrato de transferencia de tecnología de la Universidad a la EBT se debe suscribir un contrato de socios, que contengan cláusulas para que la Universidad salvaguarde sus intereses y limite sus riesgos por participar en el proyecto empresarial (derecho de veto para determinados supuestos, derecho de acompañamiento, derecho de mejor fortuna, derecho antidilución, derecho de salida de la EBT en determinados supuestos, derecho de auditoría por parte de la Universidad, etc.). Las actuales reticencias de algunas Universidades españolas a ser socias de las EBT que se constituyen en su entorno (miedo a la responsabilidad por concurso de la sociedad, imagen excesivamente mercantilista de la Universidad, complicaciones organizativas, etc.) deberán, con las debidas cautelas, superarse.

Esta participación en el capital social puede ser in natura (patentes, un saber hacer, derecho de uso de instalaciones, arrendamiento, etc. ${ }^{11}$ ) o, lo que está mucho más generalizado por su sencillez, dineraria. La aportación al capital social de la Universidad puede realizarse en un momento inicial de constitución de la empresa o en un momento posterior en una ampliación de capital de la sociedad ya constituida (algo que por el tenor literal de la disposición adicional 24. ${ }^{\text {a }}$ parecía no permitirse para levantar las incompatibilidades comentadas). Si la Universidad participa en el capital social de forma mayoritaria, se le aplica el régimen de las empresas públicas y queda sometida a las obligaciones de rendir cuentas propias de la Universidad (art. 84 LOMLOU). No obstante, lo habitual es que la participación de las Universidades no sea superior al $10 \%$ del capital social en sociedades anónimas o de responsabilidad limitada, con lo que las responsabilidades por pérdidas o concurso, quedan limitada a dicha aportación.

Por otra parte, gran parte de las ventajas que pueden tener los investigadores que constituyan una spin-off académica siguiendo el largo proceso de constitución que han articulado las distintas universidades, y no participar sin más en un proyecto empresarial al margen de la Universidad (cosa que pueden hacer siempre que no sobrepasen el $10 \%$ del capital social cuando la empresa va a tener un ámbito de actuación similar a la que realiza a la Universidad, y si es de un ámbito distinto no tiene límites), es la posibilidad de regularizar su situación en la empresa, tanto en la articulación de la transferencia de tecnología desde la Universidad a la empresa como en la legalidad de su actuación empresarial, sin contar con el

\footnotetext{
11 BERCOVITZ ÁllVAREZ, R. La aportación de Derechos de Propiedad Industrial al capital de las Sociedades Anónimas. Madrid, 1999, p. 82 y s.
} 
interés curricular cada vez más valorado de ser socio de una EBT como indicador de transferencia. Pero si quiere que le autoricen a trabajar en la empresa, pedir una excedencia o poder tener más de un $10 \%$ del capital social y ser miembro del órgano de administración, la Ley exige que la Universidad participe como socio en el proyecto empresarial.

Esta participación de la institución en el capital social se ve más fácil en una sociedad de responsabilidad limitada o en una sociedad anónima que en una sociedad laboral o en una cooperativa. Y aunque es cierto que en ambos casos se permite que la administración pública participe como socio capitalista, colaborador o asociado, son situaciones complejas por su excepcionalidad, las Universidades, preocupadas por no tomar mayores riesgos que los absolutamente necesarios a la hora de convertirse en socios de spin-offs van a preferir simples sociedades de capital y no empresas de economía social. Por ejemplo, pensando ahora en las estipulaciones de salvaguarda que se deben incluir en los acuerdos de socios, para que la insitución pueda salirse en cualquier momento del accionariado, están pensadas para sociedades mercantiles típicas (antidilución, mejor fortuna, etc.) en las que la posibilidad de transmisión de la posición de socio está sumamente facilitada.

Eso no quita, que se pueda organizar la spin-off como una empresa de economía social de hecho pero no de Derecho, y cumplir voluntariamente con todos o con algunos de los principios de economía social descritos (por ejemplo, con la participación de los socios en el proceso productivo, con políticas de responsabilidad social corporativa, toma de decisiones democráticas, repartos de beneficios en función de la actividad desarrollada por los socios, etc.).

\section{BIBLIOGRAFÍA}

BERCOVITZ ÁLVAREZ, R. La aportación de Derechos de Propiedad Industrial al capital de las Sociedades Anónimas. Madrid, 1999

CAYUELA LÓPEZ, M.J. y PLANAS YANES, G. Comentario art. 41. En Un paseo por la LOU. Análisis sistemático de la Ley Orgánica 6/2001, de 21 de diciembre de Universidades. Madrid, 2003, p. 390 y ss.

COSTAS DEL PORTAL, I. y OURO FUENTE, A. Participación de los investigadores en los resultados de la investigación. El entorno legal: proyectos de Ley de la Ciencia, la 
Tecnología y la Innovación y de la Ley de Economía Sostenible. La Ley, n 7615, 2011, p. $1-15$.

CRUE. Informe Universidad 2000. Madrid: CRUE, 2000.

GÓMEZ OTERO, C. y VALLE PASCUAL, J.M. Si el legislador nos echara una mano. En $I V$ Seminario sobre aspectos jurídicos de la gestión universitaria. Girona, 2001.

KRIMSKY, S. Biotechnics and Society: The Rise of Industrial Genetics. New York: Praege, 1991.

LEYDESDORFF, H. y ETZKOWITZ, H. Emergence of a Triple Helix of UniversityIndustry-Government Relations. Science and Public Policy, 1996, p. 42 y ss.

MELIÁN NAVARRO, A. y CAMPOS CLIMENT, V. Emprendedurismo y economía social como mecanismo de inserción sociolaboral en tiempos de crisis. REVESCO, $\mathrm{n}^{\circ} 100,2010$, p. $46-67$.

MONOTTI, A. y RICKETSON, S. Universities and Intellectual Property. Ownership and Exploitation. Oxforf, 2003.

PANIAGUA ZURERA, M. Las empresas de la economía social más allá del comentario a la Ley 5/2011, de economía social. Madrid: Marcial Pons, 2011.

PETIT LAVALL, M. ${ }^{a}$ V. El anteproyecto de Ley de Economía Sostenible y la transferencia de resultados de la actividad investigadora de las Universidades públicas mediante la constitución de Empresas de Base Tecnológica (EBTS). En GÓMEZ-SEGADE y GARCÍA VIDAL. El Derecho Mercantil en el umbral del siglo XXI. Libro homenaje Fernández-Novo. Madrid, 2010.

SlAUgther, S. y LESLIE, L.L. Academic Capitalist. Politics, Policies and the Entrepreneurial University. Baltimore: The Johns Hopkins, 1997.

SOSA WAGNER, F. El mito de la autonomía universitaria. Madrid, 2007.

VALLE PASCUAL, J.M. y MARTÍNEZ FERNÁNDEZ, S. La transferencia del conocimiento, ¿una nueva función social universitaria? Medios personales y materiales. En Bases jurídicas para la gestión universitaria, t. I. Alicante: Universidad de Alicante, 2008, p. 285-316.

VARGAS VASSEROT, C. La titularidad de las patentes universitarias: el caso Gatorade. Uciencia, $\mathrm{n}^{\circ} 5,2010$, p. 14 y s.

VARGAS VASSEROT, C. Las empresas de base tecnológica como instrumento de transferencia de resultados de invesrtigación. En Estudios de Derecho Mercantil en homenaje al Profesor José María Muñoz Planas. Madrid: Civitas, 2011, p. 857-876. 